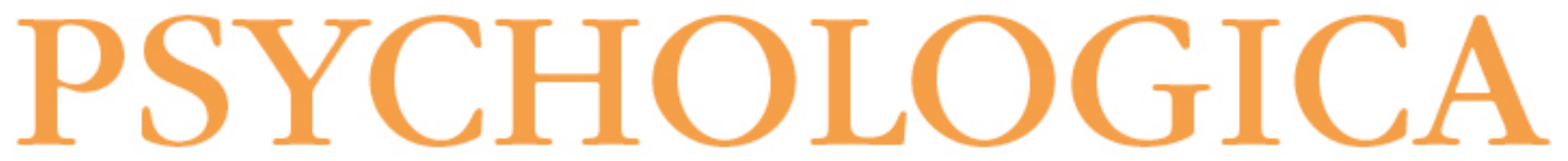

\title{
Análisis de las características de estabilidad emocional en hombres y mujeres
}

Autor(es): $\quad$ Gracia, Esther; Vidal-Sellés, Naiara; Martínez-Tur, Vicente

Publicado por: Imprensa da Universidade de Coimbra

URL

persistente:

URI:http://hdl.handle.net/10316.2/41454

DOI:

DOI:https://doi.org/10.14195/1647-8606_59_2_8

Accessed : $\quad$ 26-Apr-2023 12:04:41

A navegação consulta e descarregamento dos títulos inseridos nas Bibliotecas Digitais UC Digitalis, UC Pombalina e UC Impactum, pressupõem a aceitação plena e sem reservas dos Termos e Condições de Uso destas Bibliotecas Digitais, disponíveis em https://digitalis.uc.pt/pt-pt/termos.

Conforme exposto nos referidos Termos e Condições de Uso, o descarregamento de títulos de acesso restrito requer uma licença válida de autorização devendo o utilizador aceder ao(s) documento(s) a partir de um endereço de IP da instituição detentora da supramencionada licença.

Ao utilizador é apenas permitido o descarregamento para uso pessoal, pelo que o emprego do(s) título(s) descarregado(s) para outro fim, designadamente comercial, carece de autorização do respetivo autor ou editor da obra.

Na medida em que todas as obras da UC Digitalis se encontram protegidas pelo Código do Direito de Autor e Direitos Conexos e demais legislação aplicável, toda a cópia, parcial ou total, deste documento, nos casos em que é legalmente admitida, deverá conter ou fazer-se acompanhar por este aviso. 
VOLUME

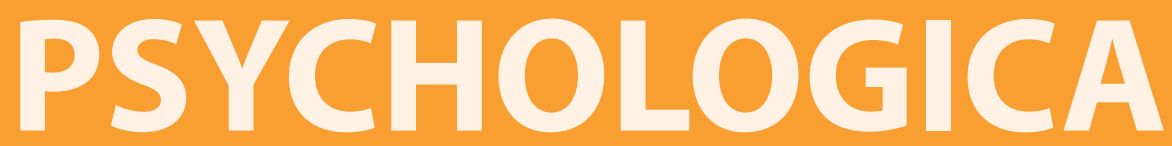

IMPRENSA DA UNIVERSIDADE DE COIMBRA

COIMBRA UNIVERSITY PRESS

FACULDADE DE PSICOLOGIA E DE CIÊNCIAS

DA EDUCAÇÃO DA UNIVERSIDADE DE COIMBRA 


\title{
Análisis de las características de estabilidad emocional en hombres y mujeres
}

\author{
Esther Gracia ${ }^{1}$, Naiara Vidal-Sellés ${ }^{2}$ y Vicente Martínez-Tur ${ }^{3}$
}

Analysis of emotional stability characteristics in men and women

\begin{abstract}
Literature points out that emotional stability, as a personality trait, affects human behaviour. This emotional stability is composed of two dimensions: The emotional disorder threshold (the easiness to experiment altered emotional states) and the emotional resilience (the ability to bounce back from negative emotional experiences to restore a stable system). The aim of this study is to test the differences between men and women in emotional stability taking into account both dimensions of emotional stability. A total 134 university students (range of age between 18 and 40 years old) participated in the study. Results showed that women have both a lower emotional disorder threshold to experience negative emotions and a lower emotional resilience than men. These results encourage continuing doing research in this line to get a better understanding of human behaviour.
\end{abstract}

Keywords: emotional stability; evolutionary theory; social role theory, men and women

1 Universidad de Valencia, Instituto de Investigación en Psicología de los Recursos Humanos, Desarrollo Organizacional y Calidad de Vida Laboral (IDOCAL). Email: esther.gracia@uv.es

2 Universidad de Valencia, Instituto de Investigación en Psicología de los Recursos Humanos, Desarrollo Organizacional y Calidad de Vida Laboral (IDOCAL). Email: naivise@alumni.uv.es

3 Universidad de Valencia, Instituto de Investigación en Psicología de los Recursos Humanos, Desarrollo Organizacional y Calidad de Vida Laboral (IDOCAL). Email: vicente.martinez-tur@uv.es

Artigo por convite recebido a 02-09-2016 e aprovado a 03-09-2016. 


\section{Resumen}

La investigación previa indica que la estabilidad emocional, como rasgo de personalidad, tiene una repercusión relevante sobre el comportamiento humano. Dicha estabilidad tiene dos dimensiones: El "umbral de alteración emocional" (facilidad para experimentar estados emocionales alterados) y la "resiliencia emocional" (la capacidad para recuperarse de las emociones negativas y volver a la estabilidad emocional). El objetivo de este estudio consiste en analizar las diferencias entre hombres y mujeres en cuanto a sus niveles de estabilidad emocional, teniendo en cuenta ambas dimensiones. La muestra está formada por 134 estudiantes universitarios de edades comprendidas entre 18 y 40 años. Los resultados muestran que las mujeres poseen tanto un umbral más bajo para experimentar emociones negativas como una menor resiliencia emocional que los hombres. Dichos datos indican la necesidad de realizar más estudios en esta dirección para llegar a comprender mejor el comportamiento del ser humano.

Palabras clave: estabilidad emocional; teoría del rol social; teoría evolucionista; hombres y mujeres

Análise das características de estabilidade emocional em homens e mulheres

\section{Resumo}

A investigação prévia indica que a estabilidade emocional, como um traço de personalidade, tem uma repercussão relevante sobre o comportamento humano. Esta estabilidade tem duas dimensões: o "limiar de distúrbio emocional" (facilidade em experimentar estados emocionais alterados) e a "resiliência emocional" (capacidade para recuperar de emoções negativas e voltar à estabilidade emocional). O objetivo deste estudo é analisar as diferenças entre homens e mulheres quanto aos seus níveis de estabilidade emocional, tendo em conta as duas dimensões de estabilidade emocional. A amostra é constituída por 134 estudantes universitários com idades compreendidas entre os 18 e os 40 anos. Os resultados mostram que as mulheres têm um limiar mais baixo para experimentar emoções negativas e uma menor resiliência emocional. Estes resultados apontam para a necessidade de se realizarem mais estudos neste domínio para uma melhor compreensão do comportamento humano.

Palavras-chave: estabilidade emocional; teoria do papel social; teoria evolucionária; homens e mulheres 


\section{INTRODUCCIÓN}

Existe creciente evidencia de que la gestión de las emociones es fundamental para llevar una vida satisfactoria en sus diferentes ámbitos. Las publicaciones especializadas identifican constructos emocionales clave que se utilizan cada vez más tanto en la investigación como en los planes de formación personal o profesional. Este es el caso de la Inteligencia Emocional, considerada como el conjunto de habilidades que contribuyen a un funcionamiento psicológico y social óptimo a través de la detección, el entendimiento y la gestión de los estados emocionales, y que facilitan la interacción adecuada con otras personas de nuestro entorno (Brackett, Rivers, \& Salovey, 2011). Otro ejemplo es el Trabajo Emocional, que implica mostrar la emoción apropiada en el contexto laboral de acuerdo con una regla implícita o explícita socialmente aceptada, entre otros aspectos. Este trabajo emocional, y la regulación emocional subyacente, afectan tanto al bienestar como al desempeño del trabajador (Gracia, Ramos, \& Moliner, 2015). En este conglomerado de constructos de naturaleza emocional, la estabilidad emocional adquiere cada vez mayor interés. Entendida como rasgo de personalidad, la estabilidad emocional describe disposiciones relativamente estables de las personas en relación con la capacidad, ante una situación emocionalmente inestable, de mantenerse o volver a un estado de equilibrio, de manera automática y recursiva, sin haber operado ninguna fuerza externa (Li, Chun, Ashkanasy, \& Ahlstrom, 2012). En otras palabras, existen diferencias en el grado en que las personas volvemos a un estado de equilibrio tras experimentar una situación emocionalmente inestable. Es decir, los individuos varían en su capacidad para mantener su estabilidad psicológica al hacer frente a las presiones difíciles del contexto (Li \& Ahlstrom, 2016).

La investigación lleva una larga trayectoria demostrando la importancia de la estabilidad emocional en diferentes áreas. Se ha relacionado con cuestiones tan relevantes como el éxito académico (Flemming, 1928; Keys \& Whiteside, 1930), un menor consumo de drogas especialmente en hombres jóvenes (Steffenhagen, Schmidt, \& McAree, 1971), la habilidad para la resolución de problemas (Park \& Woo, 1997) o la longevidad (una media de dos o tres años más) (Terracciano, Löckenhoff, Zonderman, Ferrucci, \& Costa, 2008). También se ha observado que la estabilidad emocional se relaciona, en el ámbito laboral, con el liderazgo emergente, la satisfacción, la autoeficacia y el compromiso organizacional (Li \& Ahlstrom, 2016). Concretamente, la estabilidad emocional parece tener un papel clave en situaciones de conflicto. Por ejemplo, Li et al. (2012) encontraron que el liderazgo emergente se daba en aquellas personas que recuperaban su estabilidad emocional de forma más fácil y rápida, y sobre todo en grupos conflictivos. En la misma línea, se ha comprobado que la estabilidad emocional amortigua la relación negativa entre el conflicto de relación grupal y el compromiso organizacional del individuo (Li \& Ahlstrom, 2016). 
La estabilidad emocional se ha abordado desde la Teoría de la Auto-organización (Izard, Ackerman, Schoff, \& Fine, 2000). Así, se asume que una emoción puede recuperar su estado de equilibrio automáticamente tras un periodo o situación de inestabilidad emocional. Esta disposición de las personas para adaptarse a los estresores está compuesta por dos dimensiones o subsistemas: El umbral de alteración emocional y la resiliencia emocional (Li \& Ahlstrom, 2016). Por un lado, el umbral de alteración emocional describe la sensibilidad del sistema para experimentar estados emocionales alterados como el enfado, la ansiedad o el pánico. Por ello, se espera que una persona con un bajo umbral de alteración emocional se vea afectada más fácilmente por estados emocionales alterados producidos por el ambiente. Por otro lado, la resiliencia emocional hace referencia al tiempo requerido para recuperarse de la experiencia de emociones negativas y volver a la estabilidad. En este caso, una alta resiliencia emocional hace referencia a la capacidad del individuo para recuperarse rápidamente de la experiencia de emociones negativas (Li \& Ahlstrom, 2016).

Estudios previos han encontrado que existen diferencias significativas entre hombres y mujeres en cuanto a niveles de estabilidad emocional (Booth \& Irwin, 2011; Rossier, Quartier, Enescu, \& Iselin, 2007; Vecchione, Alessandri, Barbaranelli, \& Caprara, 2012). Sin embargo, hasta la fecha no se han tenido en cuenta las dimensiones de estabilidad emocional que se plantean desde la teoría de la autoorganización. El presente trabajo da un paso más allá en el estudio de la estabilidad emocional examinando las diferencias entre hombre y mujeres teniendo en cuenta las dimensiones de umbral de alteración emocional y resiliencia emocional.

Las diferencias entre hombres y mujeres pueden explicarse desde dos grandes aproximaciones: la perspectiva evolucionista y la teoría de roles sociales (Eagly, 1987). Por un lado, desde una perspectiva evolucionista (Buss, 1991), se señala que las emociones tienen una función motivacional que da cualidades adaptativas relevantes a las emociones para muchas cuestiones. Por ejemplo, el miedo nos protege de agresiones físicas o verbales y la culpa nos lleva al razonamiento moral, a empatizar, a la reparación de relaciones dañadas (Izard, 2001). En general, las emociones negativas ejercen funciones de protección y guía en los dominios físicos y mentales (Izard, 2001). Desde esta perspectiva evolucionista, también se señala que los hombres y las mujeres comparten la mayoría de estructuras funcionales universales y tan solo se diferencian en aquellos dominios en los que han tenido que hacer frente en su historia evolutiva a diferentes problemas adaptativos recurrentes (Buss, 1991; Buss, 1995). Así, por ejemplo, en el contexto del estudio del conflicto se ha visto que los hombres y las mujeres reaccionan de forma diferente ante una amenaza, de tal modo que los hombres reaccionan de forma más agresiva que las mujeres (Van Vugt, De Cremer, \& Janssen, 2007).

Por otro lado, la teoría del Rol Social (Eagly, 1987) surge con la intención de explicar las diferencias de la conducta humana entre hombres y mujeres. Desde esta 
perspectiva, se señala que son las creencias estereotipadas las que determinan las interacciones sociales del día a día, lo cual lleva, a su vez, a la diferenciación sexual del trabajo y la jerarquía de género de la sociedad (Eagly, Wood, \& Diekman, 2000). Cialdini and Trost (1998) caracterizan estas normas sociales como "cautelares o por mandato judicial". Esto es, comportamientos que las personas perciben que deben hacer, o idealmente deberían hacer, por lo que, si no se llevan a cabo, producen malestar y desaprobación. Teniendo en cuenta que los roles familiares tradicionales diferenciados enmarcan a los hombres en especialistas en la instrumentalización y a las mujeres en expresividad (Parsons \& Bales, 1955), podríamos esperar que las mujeres posean un umbral de alteración emocional más bajo que los hombres. Es decir, que ante un evento afectivo negativo se espera una mayor expresividad emocional entre las mujeres para actuar de acuerdo con lo que socialmente se espera de ellas. Por ello, se plantea la siguiente hipótesis:

Hipótesis 1. Las mujeres presentan una mayor facilidad para responder a un estímulo emocional negativo. Es decir, poseen un umbral de alteración emocional significativamente más bajo que los hombres.

En una misma línea, se ha visto que en grupos mixtos (compuestos por hombres y mujeres), los hombres se orientan más a la consecución de objetivos mientras que las mujeres se ocupan más del mantenimiento del grupo y de los posibles problemas o preocupaciones sociales (Strodtbeck \& Mann, 1956). Con lo cual, las reacciones de las mujeres deben ser más persistentes antes pérdidas y agresiones que afecten a sus allegados. De este modo, es razonable pensar que, ante experiencias emocionales negativas, sea más difícil para las mujeres volver al estado inicial de equilibrio, y por ello la recuperación sea más lenta. Por ello, es probable que las mujeres sean capaces de mantener por más tiempo este estado negativo. Teniendo en cuenta estos argumentos, se plantea la siguiente hipótesis:

Hipótesis 2. Las mujeres poseen una capacidad de resiliencia emocional significativamente más baja que los hombres.

\section{MÉTODO}

\section{Muestra}

El presente estudio se ha llevado a cabo siguiendo los protocolos establecidos por el Comité de Ética de la Universidad de Valencia. Un total de 134 personas participaron 
voluntariamente en el estudio. Todos ellos son estudiantes con una edad media de 21.61 años (rango 18 - 40 años). De los cuales el 46\% son hombres y el 54\% son mujeres.

\section{Variables}

La escala de estabilidad emocional (ver Tabla 1) propuesta por Li et al. (2012) y validada por Li y Ahlstrom (2016) se compone de dos dimensiones:

Umbral de alteración o trastorno emocional. Esta dimensión se compone de cuatro ítems en los que a los participantes se les pregunta si experimentan emociones negativas fácilmente. La escala de respuesta es tipo Likert de 1 a 6 (totalmente en desacuerdo/totalmente de acuerdo). Un ejemplo de ítem es “ ¿Sueles sentirte ansioso fácilmente? El coeficiente Alpha de Cronbach de esta dimensión es de .73.

Resiliencia emocional. Se compone de seis ítems que miden si la recuperación tras experimentar emociones negativas es rápida. La escala de respuesta es tipo Likert de 1 a 6 (totalmente en desacuerdo/totalmente de acuerdo). Un ejemplo de ítem es “¿Sueles calmarte rápidamente después de una situación ansiosa y no verte influido por ella? El coeficiente Alpha de Cronbach de esta dimensión es de .85.

Tabla 1

Ítems de los Subsistemas o Dimensiones de la Estabilidad Emocional

\begin{tabular}{|c|c|}
\hline Subsistema & Ítem \\
\hline \multirow{4}{*}{$\begin{array}{l}\text { Umbral de } \\
\text { alteración } \\
\text { emocional }\end{array}$} & $\begin{array}{l}1 \text { ¿Sueles sentir fácilmente como si estuvieses haciendo frente a un desastre } \\
\text { inminente? }\end{array}$ \\
\hline & 2 ¿Sueles sentir pánico, miedo o sentirte asustado fácilmente? \\
\hline & 3 ¿Sueles sentirte ansioso fácilmente? \\
\hline & 4 ¿Sueles sentirte enfadado fácilmente? \\
\hline \multirow[t]{6}{*}{$\begin{array}{l}\text { Resiliencia } \\
\text { emocional }\end{array}$} & $\begin{array}{l}5 \text { ¿Sueles recuperarte del sentimiento de infelicidad rápidamente y no verte } \\
\text { influido por ella? }\end{array}$ \\
\hline & $\begin{array}{l}6 \text { ¿Sueles calmarte rápido después de una situación ansiosa y no verte influido } \\
\text { por ella? }\end{array}$ \\
\hline & $\begin{array}{l}7 \text { ¿Sueles recuperarte del sentimiento de enfado rápidamente y no verte in- } \\
\text { fluido por ella? }\end{array}$ \\
\hline & 8 ¿Sueles recuperarte del pánico o miedo rápidamente? \\
\hline & 9 ¿Sueles recuperarte de emociones negativas rápidamente? \\
\hline & 10 ¿Sueles recuperarte del estrés o la preocupación rápidamente? \\
\hline
\end{tabular}

\section{Análisis de datos}

En primer lugar, se han realizado análisis descriptivos como frecuencias, medias y desviación típicas para analizar las propiedades de la muestra y se 
ha analizado la estructura factorial de la variable de estabilidad emocional mediante un análisis factorial confirmatorio (AFC) con el programa Amos (Arbuckle, 2006).

En segundo lugar, para poner a prueba nuestras hipótesis, se ha llevado a cabo una Prueba $t$ de Student para muestras independientes, que nos permite comparar las diferencias entre los dos grupos: hombre vs. mujeres. Previamente, se han analizado las varianzas mediante la prueba de Levene que nos permite determinar si podemos asumir que las varianzas entre los grupos son iguales.

\section{RESULTADOS}

En la tabla 2 pueden observarse las medias y desviaciones típicas teniendo en cuenta a las mujeres y hombres por separado (Ver tabla 2).

Tabla 2

Descriptivos Atendiendo a la Variable Sexo

\begin{tabular}{clccc}
\hline \multicolumn{1}{c}{ Subsistema } & Sexo & $\mathrm{n}$ & Media & Desviación Típica \\
\hline Umbral de alteración emocional & Hombre & 62 & 2.41 & 0.89 \\
& Mujer & 72 & 2.85 & 1.06 \\
Resiliencia emocional & Hombre & 62 & 4.11 & 0.97 \\
& Mujer & 72 & 3.41 & 1.03 \\
\hline
\end{tabular}

La Figura 1 muestra los resultados del AFC para la estabilidad emocional. Como puede observarse, los coeficientes presentan un buen ajuste de los datos $\left(\chi^{2}(34)=52.785, p=.021 ; \mathrm{RMSEA}=.06 ; \mathrm{CFI}=.96, \mathrm{IFI}=.96, \mathrm{TLI}=.95\right)$, indicando efectivamente la existencia de las dos dimensiones o subsistemas de estabilidad emocional. Cabe tener en cuenta que el estadístico $\chi^{2}$ / G. L. presenta una alta sensibilidad al tamaño de muestra. Para muestras pequeñas, se produce un aumento de la probabilidad de error tipo II, mientras que para muestras grandes, hay una mayor probabilidad de error de tipo I. 


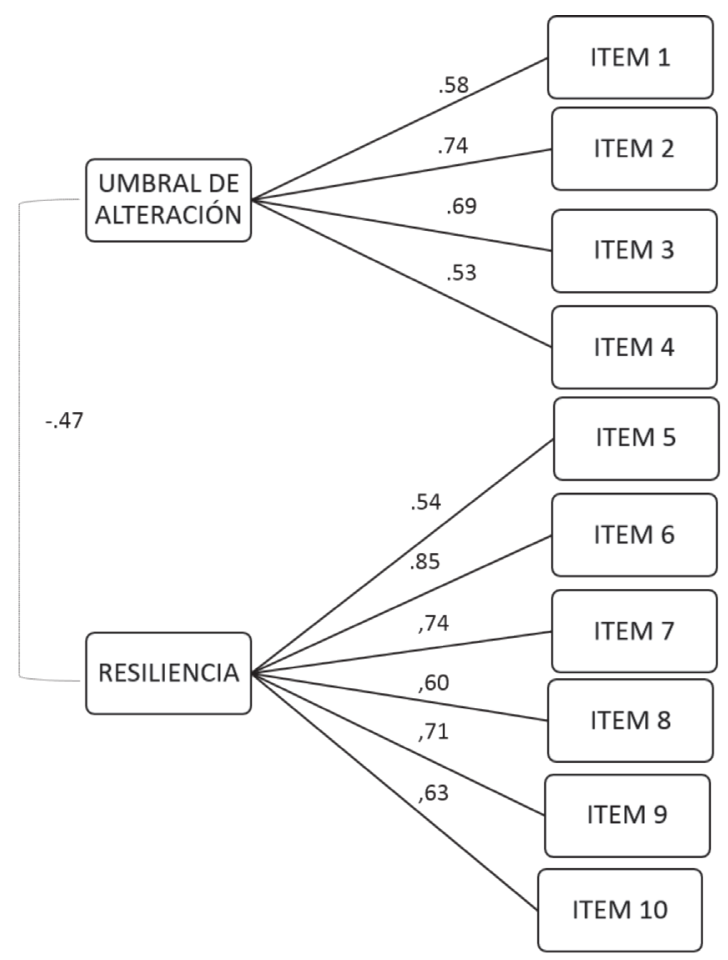

Figura 1. Análisis factorial confirmatorio de los subsistemas de estabilidad emocional.

Atendiendo a los resultados mostrados en la Tabla 3, la prueba de Levene confirma que podemos asumir varianzas iguales entre ambos grupos. Por otro lado, la prueba t de Student muestra que las diferencias son significativas tanto en el umbral de alteración emocional [ $\mathrm{t}(132)=-2.587, \mathrm{p}<.011)$ ] como en la resiliencia emocional. $[\mathrm{t}(132)=-4.046, \mathrm{p}<.000)]$

Tabla 3

Prueba de Levene y Prueba t de Student

\begin{tabular}{|c|c|c|c|c|c|c|c|}
\hline \multicolumn{2}{|c|}{$\begin{array}{l}\text { Prueba de Lev- } \\
\text { ene de igualdad } \\
\text { de varianzas }\end{array}$} & \multicolumn{6}{|c|}{ Prueba t para la igualdad de medias } \\
\hline \multirow[t]{2}{*}{$\mathrm{F}$} & Sig. & $\mathrm{t}$ & g.l. & $\begin{array}{l}\text { Sig. (bi- } \\
\text { lateral) }\end{array}$ & $\begin{array}{l}\text { Diferencia } \\
\text { de medias }\end{array}$ & $\begin{array}{l}\text { Diferencia } \\
\text { de error } \\
\text { estándar }\end{array}$ & $\begin{array}{l}95 \% \text { de intervalo } \\
\text { de confianza de la } \\
\text { diferencia }\end{array}$ \\
\hline & & & & & & & Inferior Superior \\
\hline
\end{tabular}

Umbral de alteración

$$
2.617
$$
.108 $\begin{array}{lll}-2,587 \quad 132 & 0.011\end{array}$ $-0.443$

0.171

$-.782$

$-0.104$ emocional Resiliencia emocional $\begin{array}{lllll}0.008 & .929 & 4,046 & 132 & 0.000\end{array}$ 0.705 $0.174 \quad .360$ 1.049 
De esta forma, se confirman las diferencias significativas entre hombres y mujeres en las dos dimensiones y en la dirección esperada. Las mujeres alteran más fácilmente sus estados emocionales que los hombres, es decir, ellas poseen un umbral más bajo de tolerancia para experimentar emociones negativas (hipótesis 1). Asimismo, las mujeres tienen una menor resiliencia emocional que los hombres (hipótesis 2).

\section{DISCUSIÓN}

El objetivo de este trabajo ha sido el de analizar las diferencias entre hombres y mujeres en cuanto a las dos dimensiones de estabilidad emocional: umbral de tolerancia y resiliencia emocional. Los resultados muestran que, efectivamente, existen diferencias considerables entre ambos grupos. Aunque los roles de género son un aspecto cultural dinámico (Eagly et al., 2000), que podría cambiar especialmente ante sociedades industriales con un mayor progreso sociocultural en las normas (Costa, Terracciano, \& McCrae, 2001), los resultados siguen insistiendo en la existencia de una adaptación heterogénea de hombres y mujeres ante eventos del ambiente. Los estereotipos conformados en nuestra sociedad pueden explicar parte de nuestras reacciones emocionales. Más específicamente, pueden determinar los niveles basales de alteración y nuestro tiempo de recuperación.

Además, este trabajo contribuye al avance del estudio de las emociones diferenciando entre las dos dimensiones de estabilidad emocional: umbral de tolerancia y resiliencia emocional. Para ello, tras revisar la investigación sobre estabilidad emocional, se ha utilizado una medida de estabilidad emocional que puede integrarse en el marco teórico de la auto-organización (Izard et al., 2000), y que además presenta una fácil operacionalización y aplicación a diferentes contextos (laboral, educativo, etc.). Todo ellos nos permiten avanzar en el conocimiento teórico y también establecer estrategias de intervención más concretas y aplicadas a la sociedad actual.

\section{Limitaciones}

Este trabajo presenta una serie de limitaciones que sería recomendable tener en cuenta en futuros estudios. Los resultados deben tomarse con cautela ya que han sido obtenidos en una muestra de estudiantes. La ventaja que supone es que los hombres y mujeres que han participado comparten muchas características en común como el rango de edad o su nivel académico, lo cual permite controlar algunas variables. No obstante, futuros estudios deberían probar estos resultados 
en muestras más heterogéneas para examinar la generalización de los resultados. Otra limitación importante es que este estudio transversal no permite investigar cambios en el tiempo. Futuros estudios pueden analizar trayectorias para ver si estas diferencias se mantienen a través de todas las etapas de la vida de hombres y mujeres. Una última limitación consiste en la utilización de cuestionarios auto-cumplimentados en los que solo se evalúa la percepción subjetiva del sujeto. En estudios posteriores se podrían tener en cuenta reacciones fisiológicas que complementen estas medidas.

\section{CONCLUSIONES}

A pesar de estas limitaciones, este estudio contribuye a entender el concepto de la estabilidad emocional. En primer lugar propone una definición integrada en una perspectiva teórica, así como una operacionalización clara, sencilla y aplicada. Concretamente, este estudio pone de manifiesto diferencias significativas entre hombres y mujeres en los dos subsistemas que regulan la estabilidad emocional: el umbral de alteración emocional y la resiliencia emocional. Estos resultados nos permiten llegar a comprender mejor el comportamiento del ser humano, especialmente el relacionado con las respuestas emocionales ante estímulos negativos.

\section{REFERENCIAS}

Arbuckle, J. L. (2006). Amos (version 7.0)[Computer program]. Chicago: SpSS.

Booth, T., \& Irwing, P. (2011). Sex differences in the 16PF5, test of measurement invariance and mean differences in the US standardisation sample. Personality and Individual Differences, 50(5), 553-558. doi: 10.1016/j.paid.2010.11.026

Brackett, M. A., Rivers, S. E., \& Salovey, P. (2011). Emotional intelligence: Implications for personal, social, academic, and workplace success. Social and Personality Psychology Compass, 5(1), 88-103. doi: 10.1111/j.1751-9004.2010.00334.x

Buss, D. M. (1991). Evolutionary personality psychology. Annual Review of Psychology, 42(1), 459-491. doi: 10.1146/annurev.ps.42.020191.002331

Buss, D. M. (1995). Psychological sex differences: Origins through sexual selection. American Psychologist, 50(3), 164-1688. doi: 10.1037//0003-066X.50.3.164

Cialdini, R. B., \& Trost, M. R. (1998). Social influence: Social norms, conformity, and compliance. In D. T. Gilbert, S. T. Fiske, \& G. Lindzey (Eds.), The handbook of social psychology (Vol. 2, pp. 151-192). New York: McGraw-Hill. 
Costa, J. P., Terracciano, A., \& McCrae, R. R. (2001). Gender differences in personality traits across cultures: Robust and surprising findings. Journal of Personality and Social Psychology, 81(2), 322. doi: 10.1037//0022-3514.81.2.322

Eagly, A. H. (1987). Sex differences in social behavior: A social-role Interpretation. Hilsdale, NJ: Eribaum. Eagly, A. H., Wood, W., \& Diekman, A. B. (2000). Social role theory of sex differences and similarities: A current appraisal. In T. Eckes \& H. M. Trautner (Eds.), The developmental social psychology of gender (pp. 123-174). New York: Psychology Press.

Flemming, E. G. (1928). The predictive value of certain tests of emotional stability as applied to college freshmen. Archives of psychology, 96.

Gracia, E., Ramos, J., \& Moliner, C. (2014). El trabajo emocional desde una perspectiva clarificadora tras treinta años de investigación. Universitas Psychologica, 13(4), 1517-1529. doi: 10.11144/ javeriana.upsy13-4.

Izard, C. E. (2001). Emotional intelligence or adaptive emotions? Emotion, 1(3), 249-257. doi: 10.1037/1528-3542.1.3.249

Izard, C. E., Ackerman, B. P., Schoff, K. M., \& Fine, S. E. (2000). Self-organization of discrete emotions, emotion patterns, and emotion-cognition relations. In I. Granic \& M. D. Lewis, Emotion, development, and self-organization: Dynamic systems approaches to emotional development (pp. 15-36). Toronto: Cambridge University Press

Keys, N., \& Whiteside, G. H. (1930). The relation of nervous-emotional stability to educational achievement. Journal of Educational Psychology, 21(6), 429. doi: 10.1037/h0070518

Li, Y., \& Ahlstrom, D. (2016). Emotional stability: A new construct and its implications for individual behavior in organizations. Asia Pacific Journal of Management, 33(1), 1-28. doi: 10.1007/ s10490-015-9423-2

Li, Y., Chun, H., Ashkanasy, N. M., \& Ahlstrom, D. (2012). A multi-level study of emergent group leadership: Effects of emotional stability and group conflict. Asia Pacific Journal of Management, 29(2), 351-366. doi: 10.1007/s10490-012-9298-4

Park, K. M., \& Woo, N. H. (1997). Relationship between problem solving ability and emotional stability in preschool children. Korean Journal of Child Studies, 18(2), 267-282.

Parsons, T., \& Bales, R. F. (1955). Family, socialization and interaction process. Glencoe, IL: Free Press.

Rossier, J., Quartier, V., Enescu, R., \& Iselin, A. (2007). Validation of the French version of the Hierarchical Personality Inventory for Children (HiPIC). European Journal of Psychological Assessment, 23(2), 125-132. doi: 10.1016/j.paid.2010.11.026

Steffenhagen, R. A., Schmidt, F. E., \& McAree, C. P. (1971). Emotional stability and student drug use. Journal of Drug Education, 1(4), 347-357. doi: 10.2190/R415-UQ4G-15YQ-HJN3

Strodtbeck, F. L., \& Mann, R. D. (1956). Sex role differentiation in jury deliberations. Sociometry, 19, 3-11. Terracciano, A., Löckenhoff, C. E., Zonderman, A. B., Ferrucci, L., \& Costa Jr, P. T. (2008). Personality predictors of longevity: Activity, emotional stability, and conscientiousness. Psychosomatic medicine, 70(6), 621. doi: 10.1097/PSY.0b013e31817b9371

Van Vugt, M., De Cremer, D., \& Janssen, D. P. (2007). Gender differences in cooperation and competition the Male-Warrior hypothesis. Psychological science, 18(1), 19-23. doi: 10.1111/j.14679280.2007.01842.x

Vecchione, M., Alessandri, G., Barbaranelli, C., \& Caprara, G. (2012). Gender differences in the Big Five personality development: A longitudinal investigation from late adolescence to emerging adulthood. Personality and Individual Differences, 53(6), 740-746. doi: 10.1016/j.paid.2012.05.033 